\title{
Modeling inter-laminar failure in composite structures: illustration on an industrial case study
}

\author{
M. Bruyneel", J.-P. Delsemme and Ph. Jetteur \\ SAMTECH Belgium, Liège Science Park, \\ Rue des Chasseurs-ardennais 8, Angleur, B-4031, Belgium \\ *michael.bruyneel@samcef.com \\ F. Germain
}

SAMTECH France, ZAC Saint-Martin du Touch,

Rue de Caulet 12, Toulouse, F-31300, France

\begin{abstract}
This paper presents a solution procedure made available at an industrial level to study delamination in composites. Two approaches are presented. The first one is based on an original VCE (Virtual Crack Extension) method used to provide a quick estimate of the propagation load and the critical inter-laminar cracks, in a linear finite element analysis. The second approach relies on cohesive elements, and implies a non linear analysis. More general than the fracture mechanics (VCE) approach, the cohesive elements technique allows to provide the value of the maximum load that can be sustained by the structure, and to predict the residual strength and stiffness over the fracture process. Those two methods are first compared in a DCB test case to show that the results agree well with those from the literature or with the analytical solutions. Finally, a multidelaminated industrial test case is solved with both approaches.
\end{abstract}

Keywords: delamination, industrial test case

\section{Introduction}

Assessing the damage tolerance of composite structures is clearly a challenge. At least two numerical approaches are available to study delamination with the finite element method [1]. The first one consists in using fracture mechanics in a (possibly linear) static analysis to compute the 3 modes of the strain energy release rate $\mathrm{G}_{\mathrm{I}}, \mathrm{G}_{\mathrm{II}}$ and $\mathrm{G}_{\mathrm{III}}$ (opening, sliding and tearing modes, respectively) along the different crack fronts. The most dangerous crack and the propagation load are then predicted according to criteria such as those given in equation (1), where $G_{I c}, G_{I I c}$ and $G_{I I I c}$ are the fracture toughness for each mode. With this approach crack growth analysis remains difficult to simulate and recent references only propose specific solutions for simple $3 \mathrm{D}$ configurations with a single crack front [2-6]. 


$$
\frac{G_{I}}{G_{I c}}+\frac{G_{I I}}{G_{I I c}}+\frac{G_{I I I}}{G_{I I I c}}=1
$$

In the second approach described in [1] a damage model is assigned to some interface elements inserted between plies to represent their possible decohesion and a fracture criterion is used to decide on flaws propagation [7-10]. Using such cohesive elements in the analysis allows to estimate not only the propagation load but also to predict the failure load, the crack propagation and the residual stiffness during the fracture process in an automatic way. With this information more accurate safety margins can be assessed.

Based on these observations, pure fracture mechanics seems reliable for estimating the propagation load (possibly in a - fast - linear analysis), while cohesive elements are a natural choice for cracks growth simulations, in a non linear approach.

Modeling and solving industrial problems with such finite element approaches is certainly difficult. An efficient numerical solution procedure should be able to easily insert multiple crack fronts in a given large scale meshed structure and to efficiently manage the multiple contacts between the plies and the possible non linear material behaviors assigned to the numerous interfaces. However most of the published works in the field present solutions for simple composite structures with a small amount of delamination sites and therefore few contact conditions. The objective of this note is twofold: firstly it aims at reporting the level of complexity of an industrial problem and secondly it presents the solutions obtained with the methodologies developed in the commercial finite element code SAMCEF [11], which is an implicit code. A static formulation is used here to obtain the solutions. A specific Virtual Crack Extension method (and not the Virtual Crack Closure Technique, [2]) for computing the modes of the energy release rate is briefly presented, and different strategies for insuring a good convergence of the non linear analysis, including cohesive elements, are recalled. Those two methods are first compared in a DCB test case to show that the results agree well with those from the literature or with the analytical solutions. Finally the industrial solution procedure is tested on a multi-delaminated composite. This test case is studied because it is representative of the current industrial concerns.

\section{Solution procedures for assessing damage tolerance in composites}

\subsection{The Virtual Crack Extension (VCE) method}

The Virtual Crack Closure Technique (VCCT) is certainly the most popular method for computing the modes of the energy release rate $\left(\mathrm{G}_{\mathrm{I}}, \mathrm{G}_{\mathrm{II}}, \mathrm{G}_{\mathrm{III}}\right)$ needed for estimating the onset of delamination [2-6]. A specific Virtual Crack Extension method (VCE), based on [12], is presented here as an alternative to VCCT. This method is available in the commercial FE code used here for problems that possibly include geometric non linearity, for linear and hyper-elastic materials. In VCE the variation of the total potential energy $\pi$ with respect to an increase of the crack surface $A$ is computed as a semi-analytical sensitivity analysis for the 
current displacements field in equilibrium, and therefore requires only one structural analysis. This provides the distribution of the total energy release rate $G_{T}(2)$. This computation is carried out for each node of each crack front, by slightly ("virtually") modifying the nodal coordinates in the direction normal to the crack front, thus simulating local virtual crack advances in the crack planes (see Fig. 1).

\section{Figure 1}

In (2), $\pi_{\text {pert }}$ is related to the locally modified cracked configuration at equilibrium (Fig. 1. C2), while $\pi_{\text {init }}$ corresponds to the initial crack length (Fig. 1.C1), with identical displacements.

$$
G_{T}=-\frac{\Delta \pi}{\Delta A}=-\frac{\pi_{\text {pert }}-\pi_{\text {init }}}{\Delta A}=G_{I}+G_{I I}+G_{I I I}
$$

This total energy release rate is then distributed over the opening, shearing and sliding modes based on the relative opening energies associated with each mode as in (3). $R_{i}$ are the reactions to the crack extension computed at the crack front nodes while the relative displacements $U_{i}$ are measured in the crack axes with sensor nodes located along the crack tips close to the crack front.

$$
\begin{gathered}
G_{I}=\left(U_{1} R_{1} / \sum_{i=1}^{3} U_{i} R_{i}\right) G_{T} \quad G_{I I}=\left(U_{2} R_{2} / \sum_{i=1}^{3} U_{i} R_{i}\right) G_{T} \\
G_{I I I}=\left(U_{3} R_{3} / \sum_{i=1}^{3} U_{i} R_{i}\right) G_{T}
\end{gathered}
$$

As for VCCT, accurate results are obtained for a fine mesh in the vicinity of the crack fronts.

\subsection{The cohesive elements approach}

The polynomial constitutive law for the damaged interface developed in [7] proved reliable in providing numerical results that were in very good agreement with experimental ones. According to [7], the potential assigned to the interface elements is given by (4), where three damage variables $d_{I}, d_{I I}$ and $d_{I I I}$ are considered:

$$
E=\frac{1}{2}\left[k_{I}^{0}\left\langle\varepsilon_{33}\right\rangle_{-}^{2}+k_{I}^{0}\left(1-d_{I}\right)\left\langle\varepsilon_{33}\right\rangle_{+}^{2}+k_{I I}^{0}\left(1-d_{I I}\right) \gamma_{31}^{2}+k_{I I I}^{0}\left(1-d_{I I I}\right) \gamma_{32}^{2}\right]
$$


$k_{i}{ }^{0}$ is an undamaged stiffness. The constitutive relation corresponding to this potential is written in (5):

$$
\left\{\begin{array}{l}
\sigma_{31} \\
\sigma_{32} \\
\sigma_{33}
\end{array}\right\}=\left[\begin{array}{ccc}
k_{I I}^{0}\left(1-d_{I I}\right) & 0 & 0 \\
0 & k_{I I I}^{0}\left(1-d_{I I I}\right) & 0 \\
0 & 0 & k_{I}^{0}\left(1-d_{I}\right)
\end{array}\right]\left\{\begin{array}{l}
\gamma_{31} \\
\gamma_{32} \\
\varepsilon_{33}
\end{array}\right\}
$$

The thermodynamic forces $\mathrm{Y}_{\mathrm{i}}(i=I, I I, I I I)$ are obtained by deriving (4) with respect to $d_{i}(i=I, I I, I I I)$. For mixed loading, the damage evolution is related to the three inter-laminar fracture toughness $\mathrm{G}_{\mathrm{IC}}, \mathrm{G}_{\mathrm{IIC}}$ and $\mathrm{G}_{\mathrm{IIC}}$. The equivalent thermodynamic force $\mathrm{Y}$ takes the following form:

$$
Y=\sup _{\tau \leq t} G_{I C}\left\{\left(\frac{Y_{I}}{G_{I C}}\right)^{\alpha}+\left(\frac{Y_{I I}}{G_{I I C}}\right)^{\alpha}+\left(\frac{Y_{I I I}}{G_{I I C}}\right)^{\alpha}\right\}^{1 / \alpha}
$$

where $\alpha$ is equal to 1 in the present study. It is assumed here the three damage variables have the same evolution over the loading, and a single damage $d$ is therefore used to identify delamination. The damage variable $d$ is related to the equivalent thermodynamic force $\mathrm{Y}$ via a function $\mathrm{g}(\mathrm{Y})$, i.e. $\mathrm{d}=\mathrm{G}(\mathrm{Y})$. In [7], the function $\mathrm{g}(\mathrm{Y})$ corresponds to a polynomial cohesive law. In the commercial FE code used here the approach of [7] is extended to bi-triangular and exponential softening laws [13]. The three available laws are illustrated in Fig. 2. For the first two material models of Fig. 2, the damage appears after a linear elastic behaviour of the interface (grey regions in Fig. 2), whereas for the exponential law there is no threshold and the damage occurs immediately the interface is loaded. Initially equal to zero, the damage variable reaches a unity value when all the resistance capacity of the interface has been consumed $\left(G_{i}=G_{i c}\right.$ for pure modes, or a combination of the effects via Eq. 6 for mixed modes).

\section{Figure 2}

According to fracture mechanics considerations an important parameter of the model is surely the fracture toughness $G_{i c}$, i.e. the area under the curves of Fig. 2. For a given inter-laminar fracture toughness, increasing the available inter-laminar tension $\sigma_{i}{ }^{\max }$, while keeping a constant initial stiffness, will decrease the part linked to smooth damage propagation (i.e. the length of the cohesive zone), and will provide a rough transition between finite elements for the crack propagation, possibly leading to convergence problems of the non linear numerical scheme [14]. Contrary to the fracture mechanics approach (VCE) previously presented cohesive elements can be used with a coarser mesh at the crack front location. According to [1], at least three finite elements must be active in the process zone, i.e. where intermediate damage takes place. This means that small elements should be used when the cohesive zone is small. In practice, the maximum strength of the interface $\sigma_{\text {imax }}$ is decreased [10], and a larger cohesive zone is therefore used, together with less refined mesh. Decreasing this limiting tension 
lowers the level of the structure's failure load, but eases the numerical solution procedure. It has also been observed that defining a delay in the damage occurrence, as in [15], can also limit numerical difficulties in the solution procedure, while slightly increasing the resulting maximum load level. Finally, different strategies, described in [16], for computing the material stiffness are also available here: allowing the use of the consistent, tangent or null stiffness in the softening part of the constitutive laws. A tangent stiffness is used in the following applications.

\section{The DCB test case}

In order to demonstrate the accuracy of the two approaches a simple test case is first considered. A DCB specimen with a UD lay-up (only 0 deg. plies) and a $\mathrm{A}[++30]$ lay-up $\left(\mathrm{A}[++30]=\left[ \pm 30 / 0_{6} / 0_{6} /-30 / 30 / d\right]_{\mathrm{S}}\right.$, see ref. [3]) is studied. The applied force is equal to $10 \mathrm{~N}$. The material is $\mathrm{C} 12 \mathrm{~K} / \mathrm{R} 6376$ Graphite/epoxy prepreg (see Table 1).

The DCB width is $25 \mathrm{~mm}$ for the UD and $25.4 \mathrm{~mm}$ for the $\mathrm{A}[++30]$. The length of the specimen is $150 \mathrm{~mm} .32$ plies of $0.127 \mathrm{~mm}$ define the laminate. The crack length is $57.15 \mathrm{~mm}$. The mesh used is given in [3] and shown in Fig. 3.

\section{Table 1}

\section{Figure 3}

First order volume elements with internal modes are used. Eight elements are defined over the thickness, with a refinement at the crack propagation plane including 4 layers of $0.127 \mathrm{~mm}$. A refinement is also defined at the location of the crack front: in Fig. 3, 20 finite elements are used along $10 \mathrm{~mm}$ and $l_{e}=0.5 \mathrm{~mm} .22$ elements are used across the width, with a $1 \mathrm{~mm}$ wide section on both edges modeled with 5 elements. For the model with cohesive elements, the length of the refined region is $55 \mathrm{~mm}$ instead of $10 \mathrm{~mm}$ and 16 elements are used along the width, with the $1 \mathrm{~mm}$ wide sections on both edges modeled with 2 elements. The crack is very easily inserted in the model by selecting faces (interfaces) at given locations over the thickness and assigning them interface elements. These elements are automatically generated and double coincident nodes are created. The interface elements are then deleted, leaving a mesh with double nodes modeling the crack. The contact conditions, if needed, can be defined based on the same groups of faces and corresponding nodes. Contact elements are then defined, measuring the gap between the nodes. Linear relations and lagrangian multipliers are added to the classical set of equilibrium equations.

\subsection{Solution obtained with VCE (linear analysis)}

In Figure 4, the maximum value of the mode I energy release rate over the crack front for the UD is compared to the solution from the beam theory, given in (7). 


$$
G_{I}=\frac{12 a^{2} P^{2}}{B^{2} h^{3} E_{11}}
$$

where $\mathrm{P}$ is the external load, $\mathrm{a}$ is the crack length, $\mathrm{B}$ is the specimen width, $\mathrm{h}$ is the thickness of the cantilever arm, and $\mathrm{E}_{11}$ is the modulus of elasticity. Here, the analytical solution is $\mathrm{G}_{\mathrm{I}}=5.089 \mathrm{~J} / \mathrm{m}^{2}$.

For $l_{e}=0.5 \mathrm{~mm}$ it turns out that the difference is about $10 \%$, which seems comparable to the results obtained with VCCT for the same discretization at the crack front (see [3]). When plane strain is assumed in the 3D model, the numerical solution gets closer to the analytical reference, with a difference of less than $3 \%$ for le $=0.5 \mathrm{~mm}$. The evolution of $\mathrm{G}_{\mathrm{I}}$ along the crack front for both laminates (UD and $\mathrm{A}[++30])$ is compared in Figure 4 to the solution obtained with VCCT in [3], for a same mesh $\left(l_{e}=0.5 \mathrm{~mm}\right.$, see Fig. 3$)$. It is concluded that VCE provides results in good agreement with the references. Table 2 collects some information related to these computations. Each analysis takes less than 20 seconds on a today's computer. A detailed comparison of VCCT and VCE will be undertaken in the near future.

\subsection{Solution with the cohesive elements (non linear analysis)}

Concerning the cohesive elements approach, results in close agreement with analytical solutions are obtained for the UD, for the 3 interfacial models of Fig. 2, as illustrated in Fig. 5. Besides the numerical results, two theoretical curves are plotted in Fig. 5, obtained from the beam theory. They represent the initial stiffness of the elastic cantilever beam subjected to a concentrated load $\mathrm{P}$ at one side, and the relation between the transversal displacement $\mathrm{W}$ and the applied load $P$ for a critical crack with $G_{I}=G_{I C}$ :

$$
\begin{aligned}
& w=\frac{P a^{3}}{3 E_{11} I}: \text { theoretical curve } 1 \text { in Figure } 5 \\
& w=\frac{\left(B G_{I C} E_{11} I\right)^{1.5}}{3 E_{11} I P^{2}}: \text { theoretical curve } 2 \text { in Figure } 5
\end{aligned}
$$

with

$$
I=\frac{B h^{3}}{12}
$$

The influence of the mesh on the results is also presented in Fig. 5, for the bitriangular law. As it was the case with the VCE approach, the solution converges with a mesh refinement. It is seen that for a large range of element size $\left(l_{e}=\right.$ $0.5 \mathrm{~mm}$ to $2 \mathrm{~mm})$, reliable results are obtained. For a coarser mesh $\left(l_{e}=4 \mathrm{~mm}\right)$, oscillations appear in the solution. This effect can be damped by reducing the maximum allowable stress at the interface from $5 \mathrm{~N} / \mathrm{mm}^{2}$ to $1 \mathrm{~N} / \mathrm{mm}^{2}$ (curve for $l_{e}=4 \mathrm{~mm}$ with a lower Sigma Max). However, this lowers the level of the structure's failure load. When the maximum stress at the interface is set to $25 \mathrm{~N} / \mathrm{mm}^{2}$, together with a larger initial stiffness of $130000 \mathrm{~N} / \mathrm{mm}^{3}$ instead of $10620 \mathrm{~N} / \mathrm{mm}^{3}$ (see Table1), similar results are obtained (curve for $l_{e}=0.5 \mathrm{~mm}$ with a higher Sigma Max), however to the expand of a much larger computational effort. A reduction of the maximum stress at the interface is therefore attractive 
for a fast computational solution procedure, and yet an accurate result. Table 3 collects some information related to these computations. The order of magnitude for the CPU is a couple of hours on a today's computer for the finest mesh, and a couple of minutes for the coarsest one.

\section{Figure 4}

\section{Figure 5}

\section{The multi-delaminated test case}

The following example demonstrates the methodology presented in this paper to analyse delamination and to identify the critical cracks in a multi-delaminated composite structure. The reader will appreciate that some data and experimental results are omitted here for confidentiality reasons. For real industrial applications, suitable interface properties should be derived based on a comparison of results from virtual and physical simple prototypes such as DCB. Moreover, since numerical models are involved, the sensitivity of the solution to the mesh and to the interface material properties should be studied, but these two points are not fully covered in this short paper. The test case rather aims at reporting the level of complexity of an industrial problem, and for the reasons mentioned previously the results are therefore more qualitative than quantitative. Here the mesh densities for the VCE and the cohesive elements models, as well as the interface material properties are selected based on the conclusions of the previous study on the DCB. The material properties are given in Table 1 . In the Tsection laminated composite structure depicted in Figure 6,14 cracked surfaces are located between each ply of the skin, in the cap and between the noodle and the stiffener's web. The stacking sequences include plies oriented at $0^{\circ},+45^{\circ},-45^{\circ}$ and $90^{\circ}$. The structure is subjected to a pull test. Cracks are simply inserted in the finite element model as was explained for the DCB and the very large amount of contact conditions is considered.

\section{Figure 6}

\subsection{Solution obtained with VCE (linear analysis)}

The model includes 1249044 degrees of freedom (204922 nodes, 88928 first order volume elements with internal modes and 17794 contact elements). The mesh density is selected based on the conclusions of the DCB test: in the vicinity of the crack fronts, an average mesh size $l_{e}$ of about $0.5 \mathrm{~mm}$ is used. The solution obtained with the VCE method is presented in Fig. 7, for an imposed load of 5000 N. Cracks 52 and 55 are the most dangerous (see Figure 6). The propagation load for this linear analysis is around $3650 \mathrm{~N}$ for the criterion (1). In Figure 8, the energy release rates by modes, $\mathrm{G}_{\mathrm{I}}, \mathrm{G}_{\mathrm{II}}$ and $\mathrm{G}_{\mathrm{III}}$ for the most critical cracks are 
plotted with respect to the normalized cracks width. It is clear that, depending on the location, the cracks are subjected to modes I, II and III.

For a comparison, the results obtained with a coarser mesh (166053 degrees of freedom and an average $l_{e}$ equal to $1 \mathrm{~mm}$ in the vicinity of the crack fronts) are given in Figure 9. In this case, lower energy release rates are obtained, which are less accurate, according to the conclusions we formulated in the DCB test case.

\section{Figure 7}

\section{Figure 8}

\section{Figure 9}

\subsection{Solution with the cohesive elements (non linear analysis)}

Results obtained for two different mesh refinements are presented: $l e=3 \mathrm{~mm}$ and $l_{e}=2 \mathrm{~mm}$, where $l_{e}$ measures the average in plane length of the elements in the model. The bi-triangular cohesive law is used (see Fig. 2). The displacement of the stiffener is imposed via a Rigid Body Element defined on its upper face. Figure 10 compares the load-displacement curves for both meshes. The results presented in the Figures 11 and 12 correspond to the model with $l e=3 \mathrm{~mm}$, which includes 293332 degrees of freedom (64574 nodes, 18880 first order volume elements with internal modes, 12664 2D cohesive elements and 1221 contact elements). For this model, a propagation load of about $3950 \mathrm{~N}$ is obtained. Although a first damage appears between the noodle and the stiffener, the structure fails due to a de-cohesion between the cap and the stiffener. The maximum carrying load is $6530 \mathrm{~N}$. Additional information is provided in Table 4.

A comparison between the propagation load from both approaches (VCE and cohesive elements) is not always easy, but the numerical values obtained, together with experimental results, may provide a guideline for the designer.

\section{Figure 10}

\section{Figure 11}

\section{Figure 12}




\section{Conclusion}

Solution procedures used today at an industrial level to solve complex delamination problems in composite structures have been presented. Large scale models, with large numbers of initial cracks and contact zones characterize an industrial application. The solution consists in using fracture mechanics to estimate the critical cracks and propagation load in a linear analysis, while a more advanced cohesive elements approach predicts the damage evolution and the ultimate load, in a non linear analysis. Using such analyses remains complicated. Comparison of the numerical and experimental tests will be provided in a near future. A first comparison of results from experiment and the presented numerical solution procedure in a post-buckling analysis of a damaged stiffened composite panel is available in [17]. A detailed comparative study of the VCCT and the VCE approach will be undertaken.

\section{References}

1. Camanho, P. P.: Finite Element Modeling of Fracture in Composites: Current Status and future Developments. NAFEMS Seminar - Prediction and Modeling of Failure Using FEA. NAFEMS, Roskilde, Denmark, 2006.

2. Krüeger, R.: Virtual Crack Closure technique: History, Approach and applications. Applied Mechanics Reviews, 57, 109-143 (2004).

3. Krüeger, R., Three dimensional finite element analysis of multidirectional composite DCB, SLB and ENF specimens, ISD-Report $N^{\circ} 94 / 2$, Institute for Statics and Dynamics of Aerospace Structures, University of Stuttgart, 1994.

4. Orifici, A.C., Thomson, R.S., Degenhardt, R., Bisagni, C., and Bayandor, J.: Development of a Finite Element Analysis methodology for the propagation of Delaminations in Composite Structures. Mechanics of Composite Materials, 43(1), 9-28 (2007).

5. Xie, D., and Bigger, S.B.: Progressive Crack Growth Analysis Using Interface Element Based on the Virtual Crack Closure Technique. Finite Elements in Analysis and Design, 42(11), 977-984, (2006).

6. Krüeger, R., An Approach for Assessing Delamination Propagation Capabilities in Commercial Finite Element Codes, American Society of Composites $22^{\text {nd }}$ Annual Technical Conference, University of Washington, Seattle, WA, September 17-19, 2007.

7. Ladevèze, P., Allix, O., Douchin B., and Lévêque D., A Computational Method for damage Intensity Prediction in a Laminated Composite Structure, Computational mechanics - New Trends and Applications (Idelsohn, S., Oñate E., and Dvorkin E., eds.), CIMNE, Barcelona, Spain, 1998.

8. Bui, V.Q., Maréchal, E., and Nguyen-Dang, H. Imperfect Interlaminar Interfaces in laminated Composites: Delamination with the R-Curve Effect, Composites Science \& Technology 2000, 60, 2619-2630.

9. Pantano A., and Averill R.C.: Finite Element Interface Technology for Modeling Delamination Growth in Composite Structures. AIAA Journal, 42(6), 1252-1260, (2004).

10. Turon, A., Davila, C.G., Camanho P.P., and Costa, J. An engineering Solution for Using Coarse Meshes in the Simulation of Delamination With Cohesive Zone Model, NASA/TM-2005-213547, 2005.

11. SAMCEF, Ver. 12.1, SAMTECH s.a., Angleur, Belgium, October 2006.

12. Hellen, T.K.: On the Method of Virtual Crack Extension. International Journal for Numerical Methods in Engineering, 9, 187-207, (1975).

13. Jetteur, Ph., Modèle Matériau pour l'Elément d'Interface, SAMTECH Report RS-246, 2006.

14. Alfano, G., and Crisfield, M.A.: Finite Element Interface Models for the Delamination Analysis of laminated Composites: Mechanical and Computational Issues. International Journal for Numerical Methods in engineering, 72(2), 111-170, (2001).

15. Allix, O., Feissel, P., and Thévenet, P.: A Delay Damage Mesomodel of laminates Under Dynamic Loading: Basic Aspects and Identification Issues. Computers \& Structures, 81, 1177-1191, (2003).

16. Goyal, V.K., and Klug, J.C. Interphasic Formulation for the Prediction of Delamination, $45^{\text {th }}$ AIAA/ASME/ASCE/AHS/ASC Structures, Structural Dynamics \& Materials Conference, 19-22 April 2004, Palm Springs, California, AIAA 2004-1845.

17. Bruyneel M., Degenhardt R., and Delsemme J.P. An industrial solution to simulate post-buckling and damage. JEC Composites Magazine, 48, 38-39, (2009). 


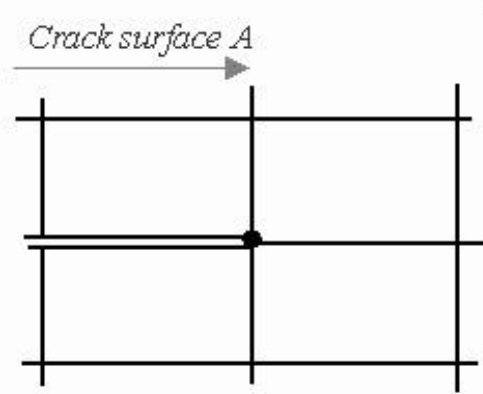

A. Modeled crack

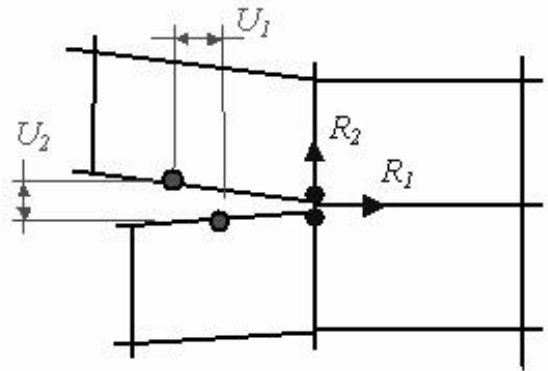

C1. Compute local forces and displacements

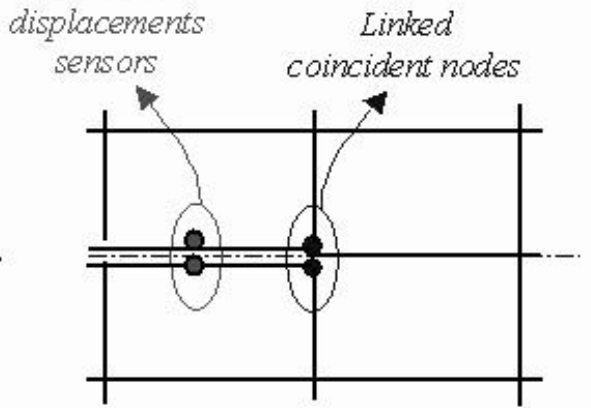

B. Computational crack

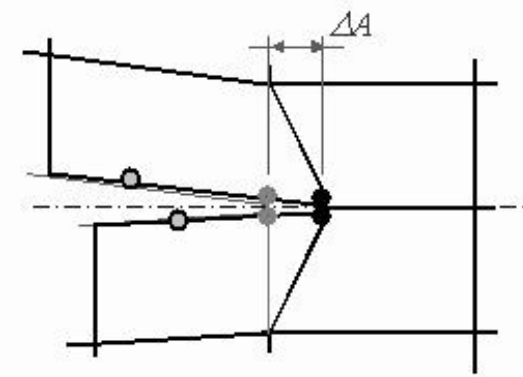

C2. Compute total energy retease rate $G_{T}$

Figure 1. Illustration of the VCE method of SAMCEF
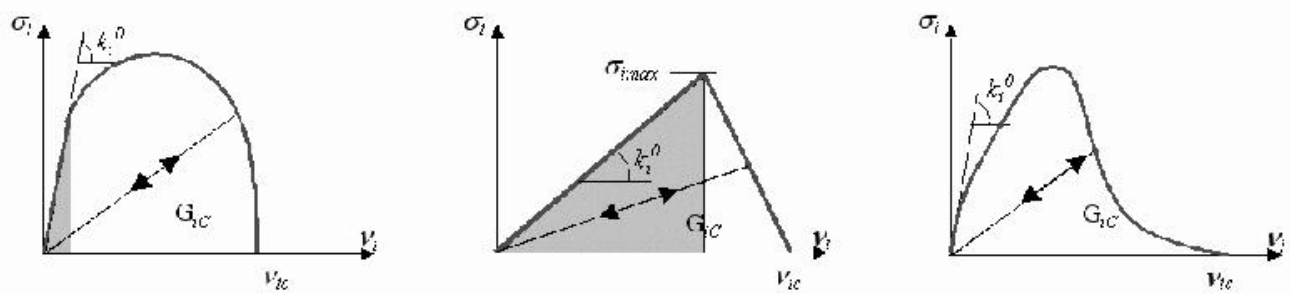

Figure 2. Material laws for the interfaces 

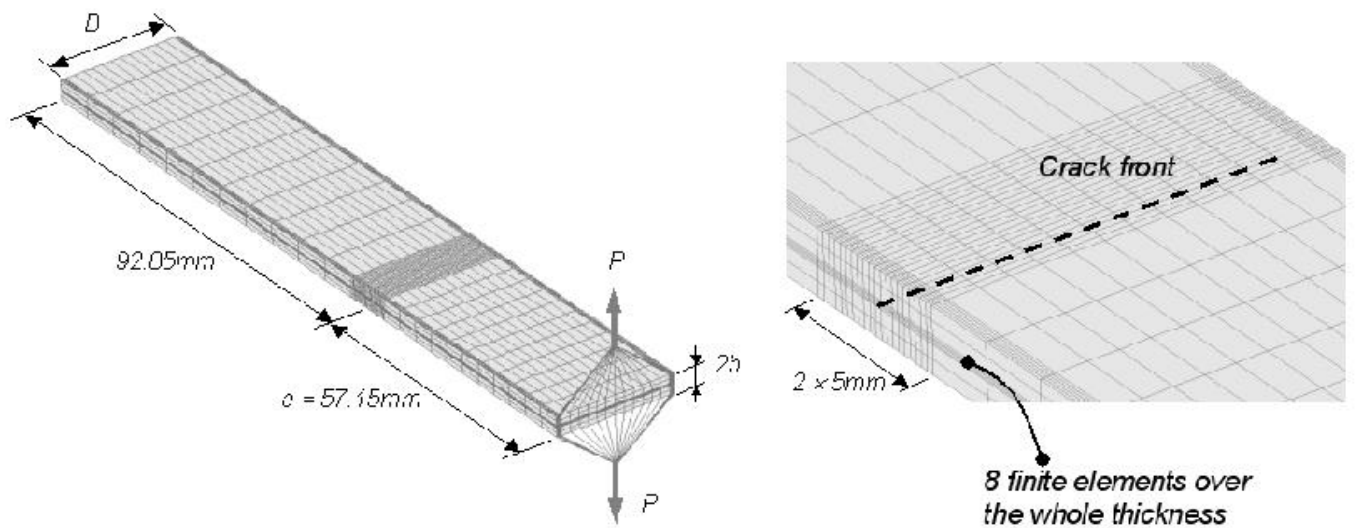

Figure 3. Mesh of the DCB, and zoom at the crack front. Here $l_{e}=0.5 \mathrm{~mm}$ (20 finite elements over a refined zone of $2 \times 5 \mathrm{~mm}$ )
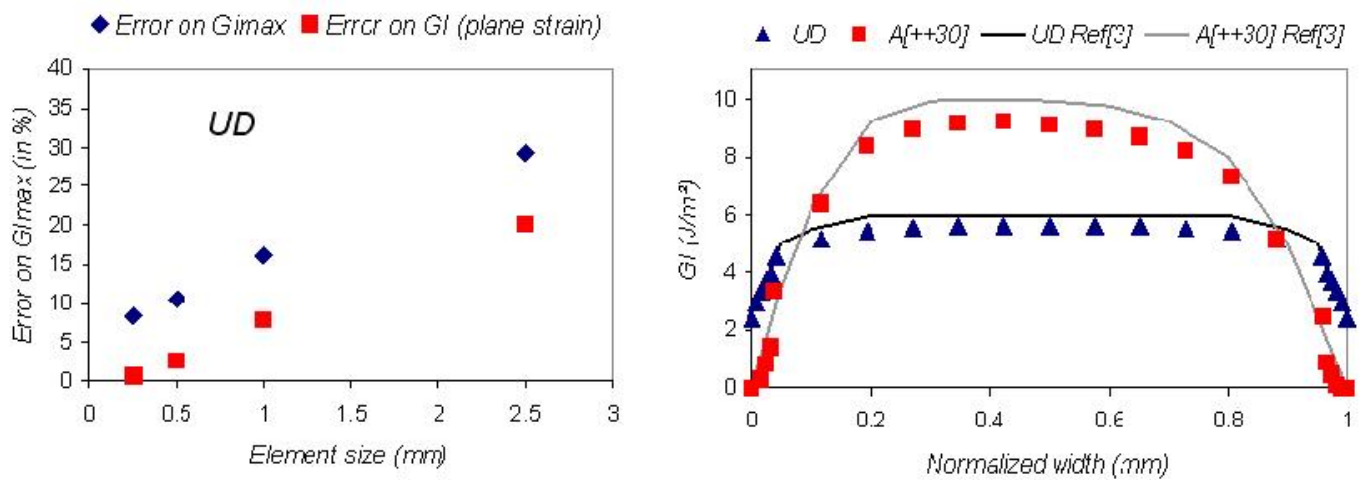

Figure 4. Results for the DCB specimen, with the VCE method. Comparison with the beam theory (on the left) and with the results from [3] (on the right) 

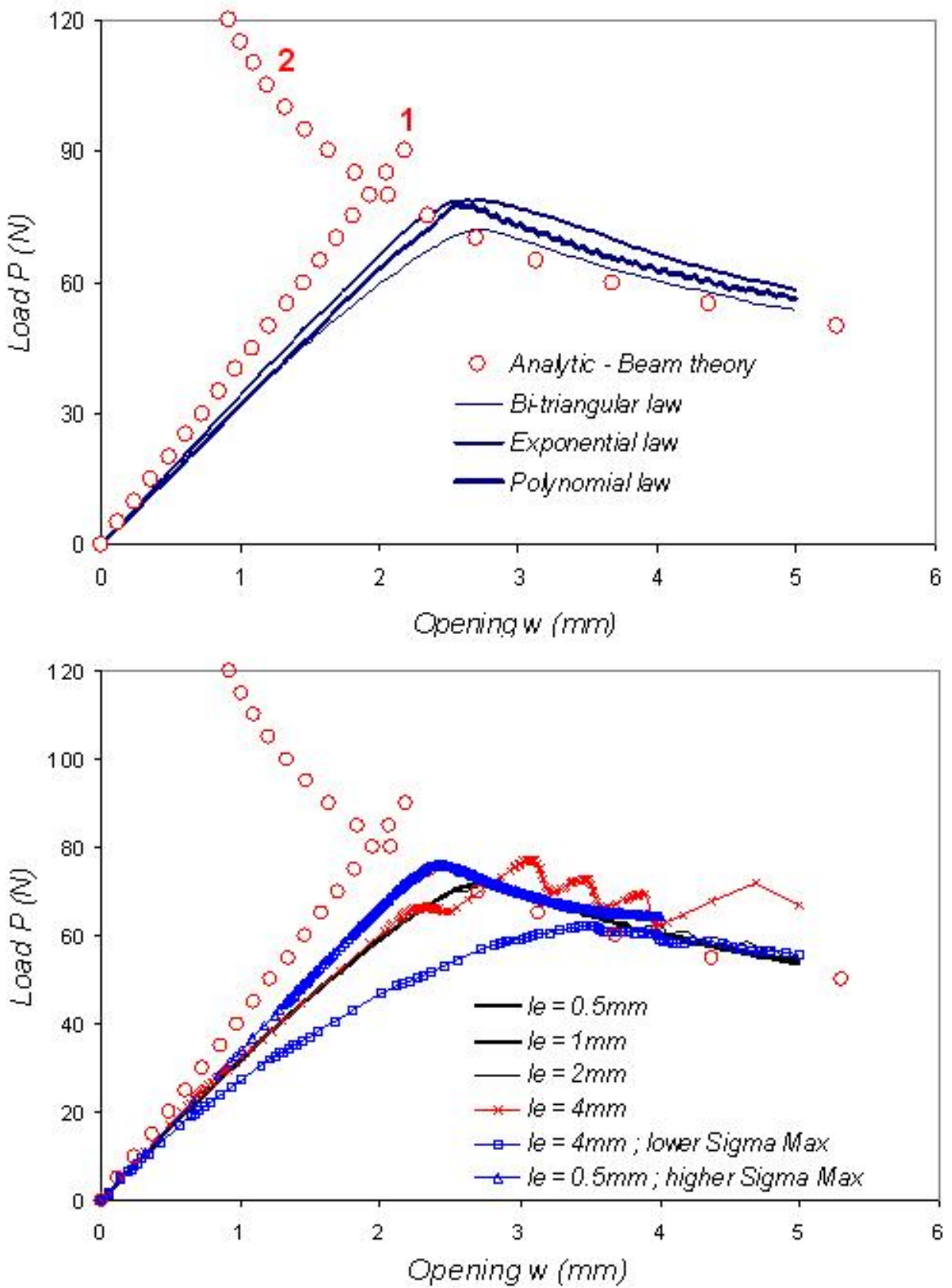

Figure 5. Results for the DCB specimen, with the cohesive elements method. On the top, comparison of the 3 available laws of Figure 2 for a mesh size $l_{e}=1 \mathrm{~mm}$. On the bottom, the influence of the mesh on the results, for the bi-triangular law. 


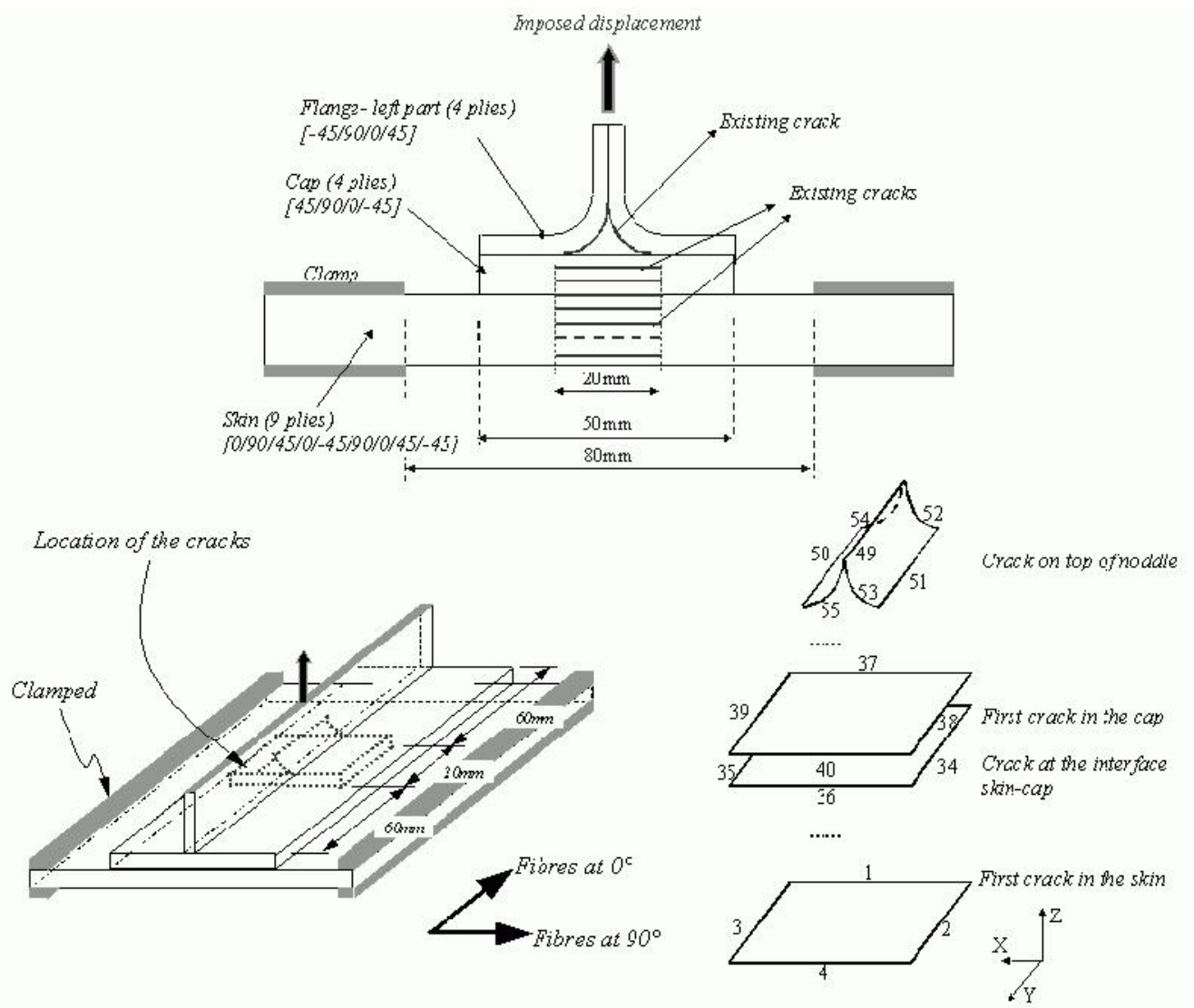

Figure 6. The laminated composite structure with multiple delamination sites subjected to a pull test. Numbering of the cracks fronts in the structure

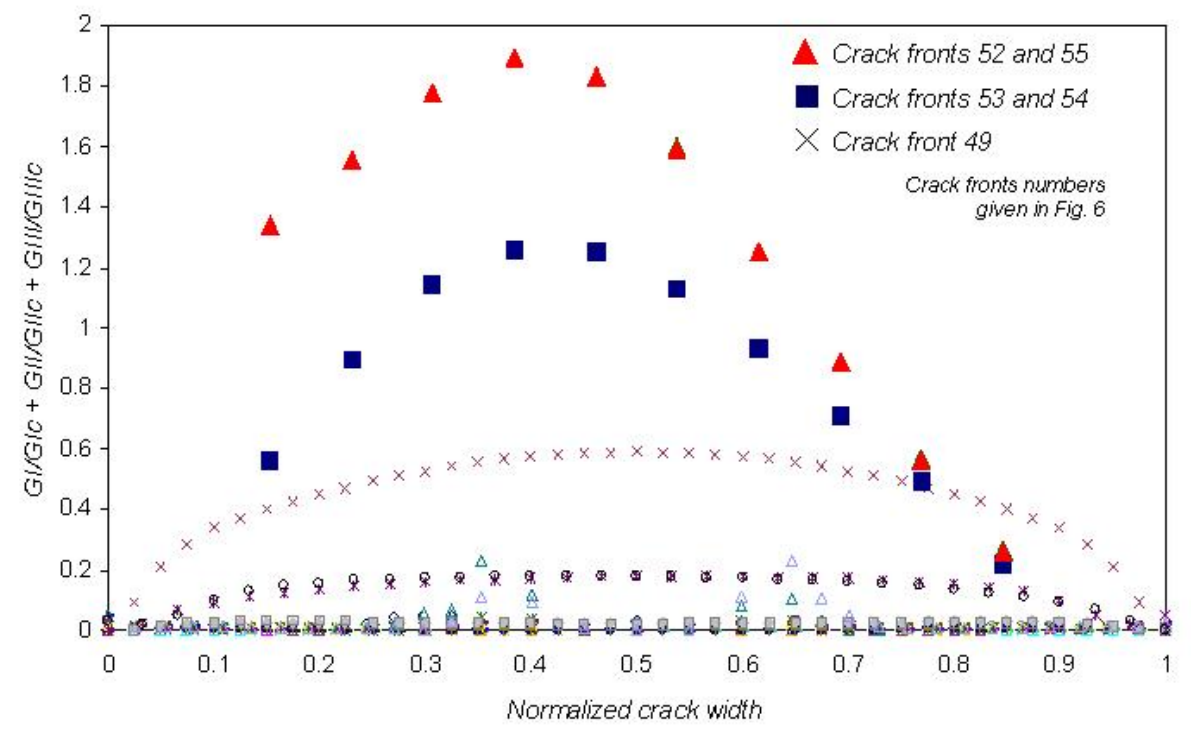

Figure 7. Evolution of the fracture criterion (1) along the crack fronts with VCE. Mesh with an average $l_{e}$ close to $0.5 \mathrm{~mm}$ 

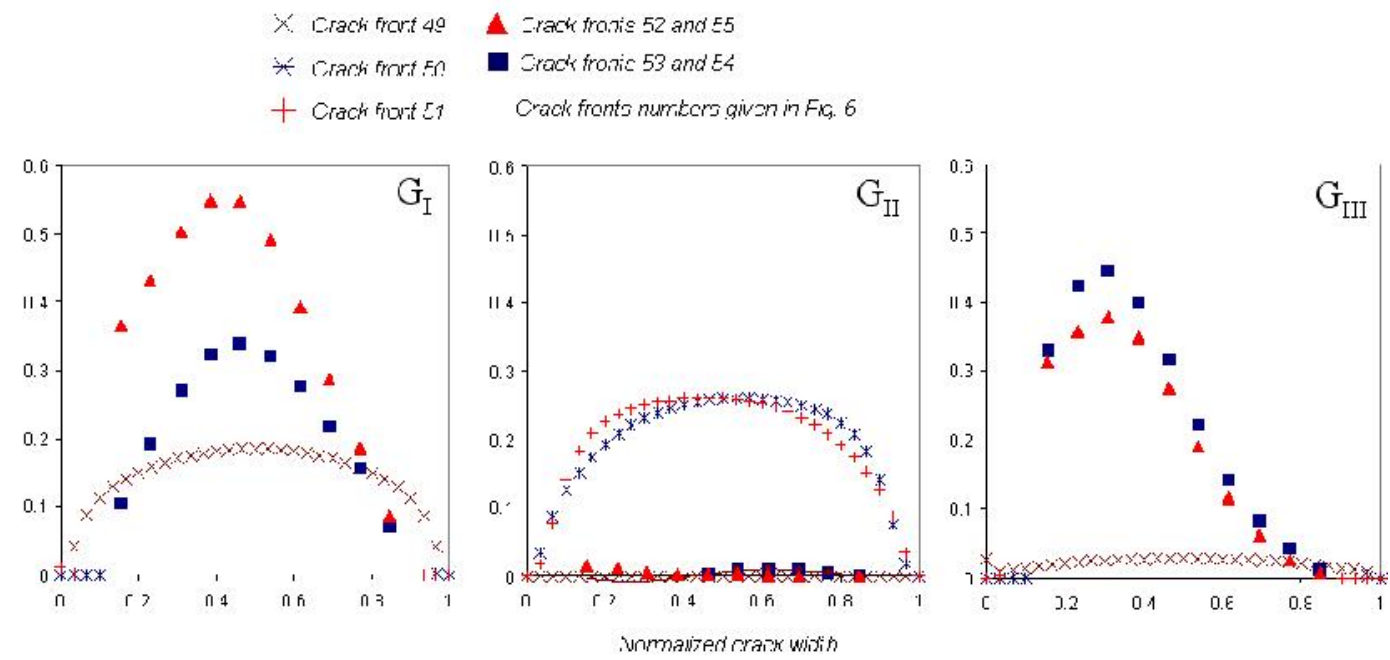

Figure 8. Evolution of the energy release rates by modes along the critical crack fronts with VCE (in $\mathrm{kJ} / \mathrm{m}^{2}$ ). Mesh with an average $l_{e}$ close to $0.5 \mathrm{~mm}$

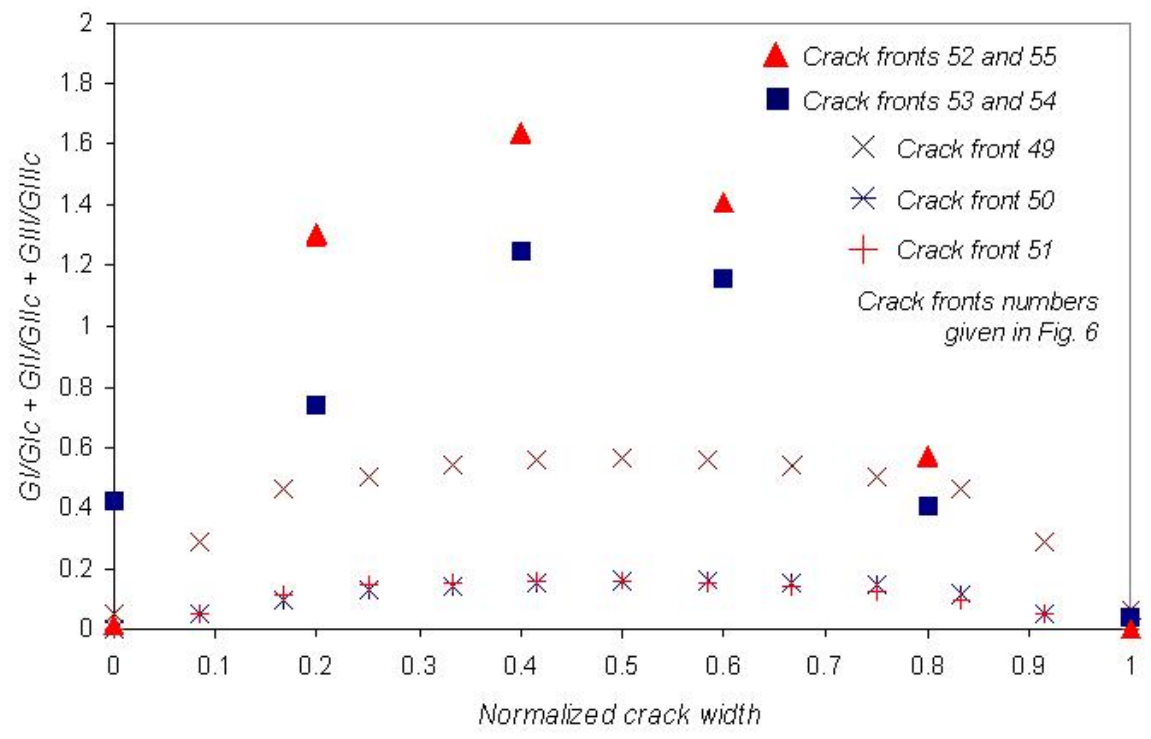

Figure 9. Evolution of the fracture criterion (1) along the critical crack fronts with VCE. Mesh with an average $l_{e}$ close to $1 \mathrm{~mm}$ 
Applied Composite Materials, 16(3), 149-162, 2009
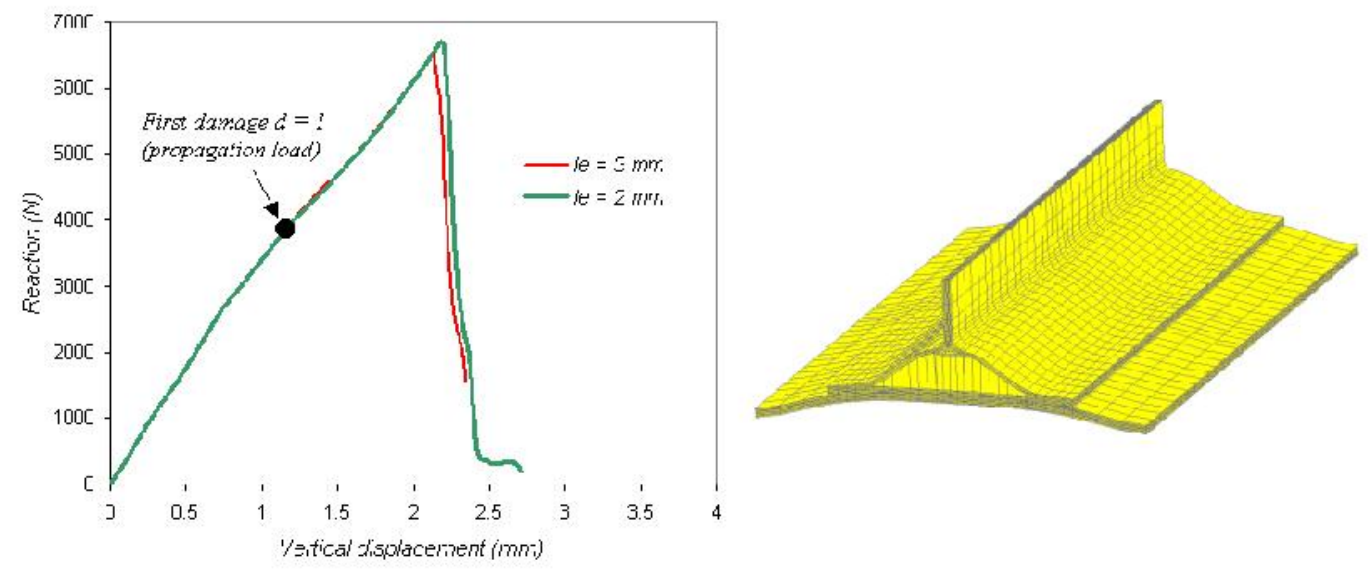

Figure 10. Load-displacement curves until failure, and displacement for $2.28 \mathrm{~mm}$ in the model with $l_{e}=3 \mathrm{~mm}$ (amplification factor for deformation $=5$ )

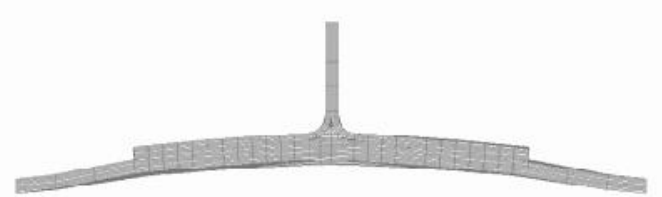

Displacement $=1.38 \mathrm{~mm}$

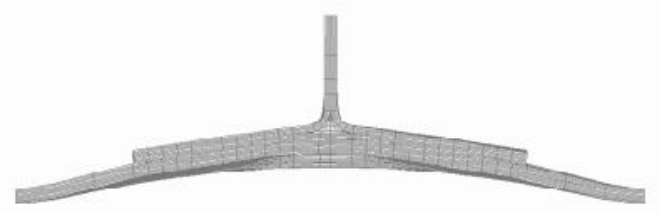

Displacement $=2.16 \mathrm{~mm}$

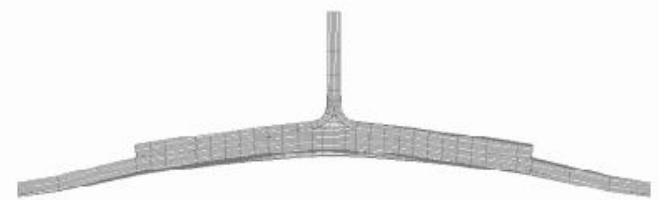

Displacement $=1.98 \mathrm{~mm}$

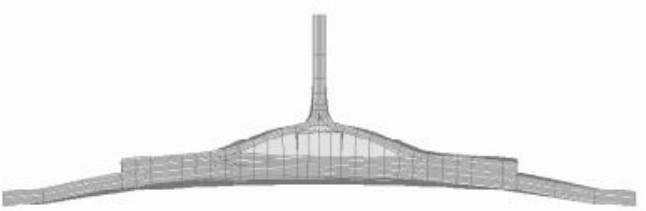

Displacement $=2.25 \mathrm{~mm}$

Figure 11. Evolution of the displacements over the loading for the model with $l_{e}=3 \mathrm{~mm}$ (amplification factor for deformations $=3$ ) 


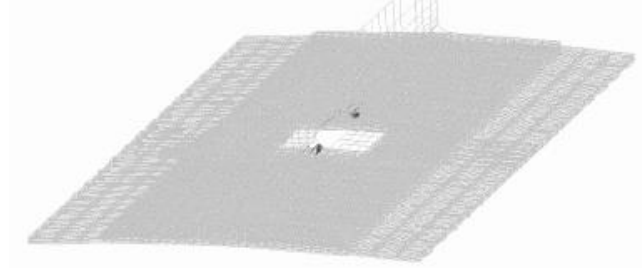

Displacement $=1.35 \mathrm{~mm}$ Load $-4450 \mathrm{~N}$

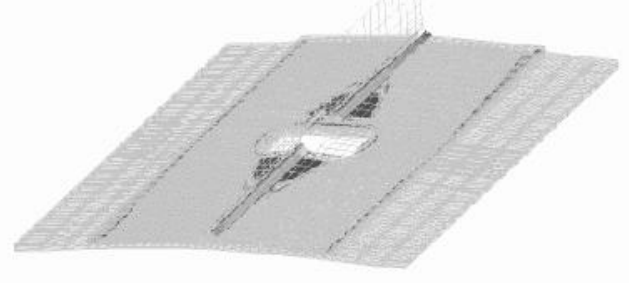

Displacement $-2.16 \mathrm{~mm}$ Load $=6100 \mathrm{~N}$

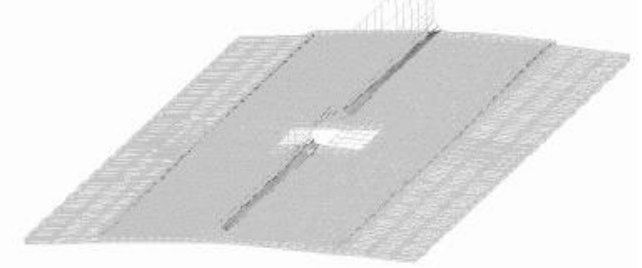

Displacement $=1.95 \mathrm{~mm}$ Load $-6050 \mathrm{~N}$

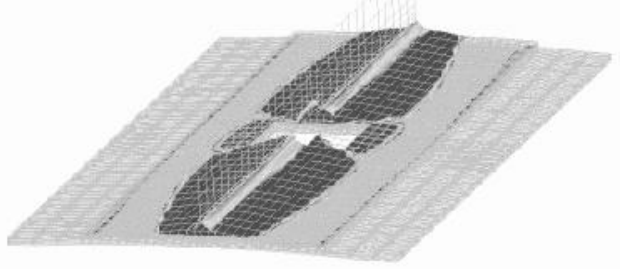

Displacement $-2.25 \mathrm{~mm}$ Load $=3000 \mathrm{~N}$

Figure 12. Evolution of the damage $(\mathrm{d} \geq 0.99)$ and displacements over the loading, up to failure, in the interface elements (model with $l_{e}=3 \mathrm{~mm}$ )

\begin{tabular}{cll}
$\mathrm{E}_{11}=146860 \mathrm{MPa}$ & $\mathrm{E}_{22}=10620 \mathrm{MPa}$ & $\mathrm{E}_{33}=10620 \mathrm{Mpa}$ \\
$v_{12}=0.33$ & $v_{13}=0.33$ & $\mathrm{v}_{23}=0.33$ \\
$\mathrm{G}_{12}=5450 \mathrm{MPa}$ & $\mathrm{G}_{13}=5450 \mathrm{MPa}$ & $\mathrm{G}_{23}=3990 \mathrm{MPa}$ \\
\hline $\mathrm{G}_{\mathrm{Ic}}=340 \mathrm{~J} / \mathrm{m}^{2}$ & $\mathrm{G}_{\mathrm{IIc}}=1280 \mathrm{~J} / \mathrm{m}^{2}$ & $\mathrm{G}_{\mathrm{III}}=1280 \mathrm{~J} / \mathrm{m}^{2}$ \\
$\mathrm{~K}_{3}^{0} \quad\left(\varepsilon_{33}\right)=10620$ & $\mathrm{k}_{1}^{0}\left(\gamma_{31}\right)=5450$ & $\mathrm{k}_{2}^{0}\left(\gamma_{32}\right)=5450$ \\
$\mathrm{~N} / \mathrm{mm}^{3}$ & $\mathrm{~N} / \mathrm{mm}^{3}$ & $\mathrm{~N} / \mathrm{mm}^{3}$
\end{tabular}

Table 1 Material properties used in the computations 
Applied Composite Materials, 16(3), 149-162, 2009

\begin{tabular}{cccc}
$\mathrm{l}_{\mathrm{e}}(\mathrm{mm})$ & Degrees of freedom & Relative CPU & $\mathrm{G}_{\mathrm{I}}$ (plane strain) $\mathrm{J} / \mathrm{m}^{2}$ \\
0.2 & & & 5.12 \\
5 & 143547 & 100 & \\
0.5 & 97377 & 60 & 5.23 \\
1 & 74292 & 44 & 5.49 \\
2.5 & 60441 & 34 & 6.12 \\
\hline
\end{tabular}

Table 2 DCB solved with the VCE approach - UD

\begin{tabular}{cccc}
\hline \hline $\mathrm{l}_{\mathrm{e}}(\mathrm{mm})$ & Degrees of freedom & Relative CPU & Maximum load (N) \\
$0.5($ higher & & 100 & 76.1 \\
$\sigma_{\text {max }}$ & 219135 & 26 & 71.9 \\
0.5 & 219135 & 10 & 71.7 \\
1 & 127593 & 6 & 72.1 \\
2 & 82515 & 4 & 77.1 \\
4 & 60909 & 2 & 62.1 \\
$4($ lower & 60909 & & \\
$\left.\sigma_{\text {max }}\right)$ & & & \\
\hline \hline
\end{tabular}

Table 3 DCB solved with the cohesive element approach - UD

\begin{tabular}{ccccc}
\hline \hline $\begin{array}{c}\text { Mean } \mathrm{l}_{\mathrm{e}} \\
(\mathrm{mm})\end{array}$ & $\begin{array}{c}\text { Degrees of } \\
\text { freedom }\end{array}$ & $\begin{array}{c}\text { Relative CPU to reach } \\
\text { the maximum load }\end{array}$ & $\begin{array}{c}\text { Maximum load } \\
(\mathrm{N})\end{array}$ & $\begin{array}{c}\text { Propagation load } \\
(\mathrm{N})\end{array}$ \\
3 & 553889 & 100 & 6700 & 3850 \\
3 & 293332 & 40 & 6530 & 3950 \\
\hline
\end{tabular}

Table 4 Multi-delamination solved with the cohesive element approach 